\title{
Functional Outcomes of Hip Arthroscopy for Pediatric and Adolescent Hip Disorders
}

\author{
Chaemoon Lim, MD, Tae-Joon Cho, MD, Chang Ho Shin, MD, In Ho Choi, MD*, Won Joon Yoo, MD \\ Division of Pediatric Orthopaedics, Seoul National University Children's Hospital, Seoul, \\ * Department of Orthopaedic Surgery, Chung-Ang University Hospital, Seoul, Korea
}

Background: There is a paucity of literature on the use of hip arthroscopy for pathologic conditions in skeletally immature patients. Thus, the indications and safety of the procedure are still unclear. The purpose of this study was to investigate the safety and functional outcomes of hip arthroscopy for pediatric and adolescent hip disorders. We further attempted to characterize arthroscopic findings in each disease.

Methods: We retrospectively reviewed 32 children and adolescents with hip disorders who underwent 34 hip arthroscopic procedures at a tertiary care children's hospital from January 2010 to December 2016. We evaluated functional limitations and improvement after operation by using the modified Harris hip score (HHS), the Western Ontario and McMaster Universities Osteoarthritis Index (WOMAC), subjective pain assessment with a visual analog scale (VAS), and range of hip motion as well as the complications of hip arthroscopy. Arthroscopic findings in each disease were recorded.

Results: Hip arthroscopy was performed for Legg-Calvé-Perthes disease $(n=6)$, developmental dysplasia of the hip $(n=6)$, slipped capital femoral epiphysis $(n=5)$, idiopathic femoroacetabular impingement $(n=6)$, sequelae of septic arthritis of the hip $(n=3)$, hereditary multiple exostosis $(n=2)$, synovial giant cell tumor $(n=3)$, idiopathic chondrolysis $(n=2)$, and posttraumatic osteonecrosis of the femoral head $(n=1)$. Overall, there was a significant improvement in the modified HHS, WOMAC, VAS, and range of hip motion. Symptom improvement was not observed for more than 18 months in four patients who had dysplastic acetabulum with a labral tear $(n=2)$ or a recurrent femoral head bump $(n=2)$. There were no complications except transient perineal numbness in five patients.

Conclusions: Our short-term follow-up evaluation shows that hip arthroscopy for pediatric and adolescent hip disorder is a less invasive and safe procedure. It appears to be effective in improving functional impairment caused by femoroacetabular impingement between the deformed femoral head and acetabulum or intra-articular focal problems in pediatric and adolescent hip disorders.

Keywords: Pediatric, Adolescent, Hip, Arthroscopy

Hip arthroscopy was first described in a cadaver study by Burman in 1931. ${ }^{1)}$ This technique has since been popularized in the diagnosis and treatment of adult hip disorders

Received March 18, 2019; Accepted July 1, 2019

Correspondence to: Won Joon Yoo, MD

Division of Pediatric Orthopaedics, Seoul National University Children's

Hospital, 101 Daehak-ro, Jongno-gu, Seoul 03080, Korea

Tel: +82-2-2072-1966, Fax: +82-2-765-3367

E-mail: yoowj@snu.ac.kr including labral tears, loose bodies, femoroacetabular impingement (FAI), ruptured ligamentum teres, chondral injuries, adhesive capsulitis, synovial diseases, and disorders of the iliopsoas tendon. ${ }^{2-4)}$ Hip arthroscopy has also been used for skeletally immature patients, because some pediatric hip disorders are complicated by FAI caused by the deformed femoral head in late disease stages and sportsrelated hip problems are increasing in this age group. The application of hip arthroscopy in children was firstly described by Gross, ${ }^{5)}$ who used the procedure for pathologic 
Lim et al. Hip Arthroscopy for Pediatric and Adolescent Hip Disorders

Clinics in Orthopedic Surgery • Vol. 12, No. 1, $2020 \bullet$ www.ecios.org

conditions such as developmental dysplasia of the hip (DDH), Legg-Calvé-Perthes disease (LCPD), slipped capital femoral epiphysis (SCFE), septic arthritis, and neuropathic subluxation of the hip. He noted that visualization of intra-articular structures was limited and orientation of the anatomy was difficult to find during the procedure. Although surgical indications for hip arthroscopy have been expanded to include some pediatric hip disorders, ${ }^{6-9)}$ there still is a paucity of literature on the outcomes of hip arthroscopy in such conditions. In this study, we aimed to investigate the safety and functional outcomes of hip arthroscopy in pediatric and adolescent hip disorders. We further attempted to characterize arthroscopic findings in each pathologic condition.

\section{METHODS}

This is a retrospective case series of 32 patients. This study was approved by the Institutional Review Board of Seoul National University Hospital (IRB No. 1810-054-977). We conducted this study in compliance with the principle of the Declaration of Helsinki. The informed consent was waived. Initially, we retrospectively reviewed 56 consecutive patients who underwent 60 hip arthroscopic procedures performed by one surgeon (WJY) at this tertiary care children's hospital from January 2010 to December 2016. All patients had pain that lasted for more than 6 months despite conservative treatment. Hip pathologies of patients were confirmed by magnetic resonance arthrograms preoperatively. Of the 56 patients, we excluded 18 patients (19 procedures) who had no impingement and who were older than 18 years and six patients (seven procedures) who were followed up for less than 18 months.

Hip arthroscopic procedures were performed in the supine position by using a fracture table for joint distraction. Anterolateral, posterolateral, and anterior portals were routinely used. Thirty- and seventy-degree arthroscopes were used and switched between portals. An arthroscopic fluid pump was maintained at $60 \mathrm{mmHg}$ and increased up to $80 \mathrm{mmHg}$ when visualization of hip joint was not optimal. Epinephrine $(1 \mathrm{mg}$ epinephrine in $3 \mathrm{~L}$ normal saline) was added to arthroscopic fluid to enhance arthroscopic visualization. Arthroscopic procedures included osteochondroplasty of the femoral bump, debridement of the torn labrum and detached articular cartilage, labral repair, removal of loose body, and synovectomy. Postoperatively, patients used crutches for three to 6 weeks. Continuous passive motion exercises were started on the second postoperative day. Patients were instructed to be in the prone position for more than 2 hours a day during the first 2 weeks after surgery.

We evaluated functional limitations and improvement after surgery by using the modified Harris hip score (HHS), the Western Ontario and McMaster Universities Osteoarthritis Index (WOMAC), subjective pain assessment with visual analog scale (VAS), and range of hip motion. All arthroscopy-related complications were reviewed. We also reviewed arthroscopic findings to see if pathologic changes in the hip joint could be characterized in each disease and to identify pathologic changes associated with poor functional outcomes. Mean values of preoperative and final follow-up evaluations were compared by using the Wilcoxon signed-rank test (SPSS ver. 18.0; SPSS Inc., Chicago, IL, USA). A p-value less than 0.05 was considered statically significant.

\section{RESULTS}

The study population was composed of 32 patients (34 procedures). There were 18 males (20 procedures) and 14 females (14 procedures). Their mean age at surgery was 14.8 years (range, 10.8 to 18.0 years). The mean follow-up period was 4.7 years (range, 2.25 to 7.6 years). Nine procedures were performed in the right hip and 25 procedures were performed in the left hip. Two patients underwent revision arthroscopy for recurrent symptoms. Specific diagnoses are as follows: $\operatorname{LCPD}(\mathrm{n}=6), \mathrm{DDH}(\mathrm{n}=6)$, SCFE $(n=5)$, idiopathic FAI $(n=6)$, sequelae of septic arthritis of the hip $(\mathrm{n}=3)$, hereditary multiple exostosis $(\mathrm{n}=2)$, synovial giant cell tumor $(\mathrm{n}=3)$, idiopathic chondrolysis ( $\mathrm{n}$ $=2$ ), and posttraumatic osteonecrosis of the femoral head $(\mathrm{n}=1)$ (Table 1).

Overall, there was a significant improvement in VAS

\section{Table 1. Specific Diagnosis of the 32 patients}

\begin{tabular}{|lc}
\hline \multicolumn{1}{|c}{ Specific diagnosis } & No. of patients \\
\hline Legg-Calvé-Perthes disease & 6 \\
\hline Developmental dysplasia of the hip & 6 \\
\hline Slipped capital femoral epiphysis & 5 \\
\hline Idiopathic femoroacetabular impingement & 6 \\
\hline Sequelae of septic arthritis of the hip & 3 \\
\hline Hereditary multiple exostosis & 2 \\
\hline Synovial giant cell tumor & 3 \\
\hline Idiopathic chondrolysis & 2 \\
\hline Posttraumatic osteonecrosis of the femoral head & 1 \\
\hline
\end{tabular}


Lim et al. Hip Arthroscopy for Pediatric and Adolescent Hip Disorders

Clinics in Orthopedic Surgery • Vol. 12, No. 1, $2020 \bullet$ www.ecios.org

from 4.4 to $2.2(p<0.001)$, with $88.2 \%$ of patients showing improvement. The modified HHS improved significantly from 77.8 to $90.0(p<0.001)$, with $85.3 \%$ of patients showing improvement. WOMAC also improved from 16.4 to 6.8 ( $p<0.001$ ), with $85.3 \%$ of patients showing improvement. The range of hip flexion improved significantly from $101.7^{\circ}$ to $115.0^{\circ}(p<0.001)$, with $97.1 \%$ of patients showing improvement (Table 2).

All patients with LCPD were in the late disease stages: reossification stage in one patient and residual stage in five. According to Stulberg classification, one hip was class II, four were class III, and one was class IV. ${ }^{10)}$ The median age at arthroscopy was 15.2 years (range, 12.3 to 17.9 years). The mean follow-up period was 5.2 years (range, 2.3 to 7.6 years). All patients showed femoral bumps and five of them also had labral tears. Four patients had ligamentum teres tears and two had osteochondritis dissecans. All patients showed symptomatic and functional improvement after arthroscopic removal of loose body, debridement of ligamentum teres and labrum, and osteochondroplasty.

In six patients with $\mathrm{DDH}$, the median age at arthroscopy was 14.6 years (range, 12.3 to 17.6 years). The mean follow-up period was 4.7 years (range, 2.8 to 7.1 years). One patient was treated with closed reduction; one patient, with open reduction; and three patients, with open reduction combined with Dega pelvic osteotomy or femoral osteotomy or both. The remaining one patient was diagnosed as having dysplastic hip at 13.3 years of age. At the time of hip arthroscopy, all patients had degenerative labral tears and femoral head deformity caused by growth disturbance of the proximal femur. Four of them also showed degenerative changes on the articular surface of the acetabulum. All patients underwent arthroscopic debridement or osteochondroplasty or both. Symptoms improved in four patients, their mean lateral center-edge angle was $22^{\circ}$ (range, $20^{\circ}$ to $24^{\circ}$ ). But two patients had a recurrence of pain at 10 months and 4 months after surgery. These two patients had dysplastic acetabulum $\left(10^{\circ}\right.$ and $11^{\circ}$ of lateral center-edge angle). They are waiting for periacetabular osteotomy.

In five patients with SCFE, the median age at arthroscopy was 15.5 years (range, 12.8 to 17.4 years). The mean follow-up period was 3.4 years (range, 2.3 to 6.2 years). The degree of epiphyseal slippage at the initial visit was mild $(n=4)$ to moderate $(n=1)$ based on Southwick angle. ${ }^{11)}$ There was no patient with severe slippage. All patients had anterolateral femoral bumps and one of them showed screw impingement against the acetabular margin. Two patients showed cartilage lesions on the anterosuperior acetabulum and labral tears. All patients showed symptomatic improvement after arthroscopic osteochondroplasty and screw removal (Fig. 1).

There were two patients (three procedures) with sequelae of septic arthritis of the hip. Their median age at arthroscopy was 14.7 years (range, 13.5 to 16.3 years). The mean follow-up period was 4.7 years (range, 3.3 to 6.3 years). Both patients showed degenerative changes in the labrum and acetabular cartilage. Pain improved immediately after labral debridement and osteochondroplasty on the impinging femoral bumps in all patients, but one patient had a recurrence of femoral bump. A revision arthroscopic osteochondroplasty and labral debridement improved pain in this patient.

Symptom improvement was not maintained for more than 18 months after arthroscopy in four patients. Three of them (two with dysplastic acetabulum and one with septic hip sequelae) are described above; the remaining one patient with multiple hereditary exostoses had a recurrent femoral bump after osteochondroplasty. Pain improved after revision arthroscopic osteochondroplasty and labral debridement. There was no serious complication related to hip arthroscopic procedures except transient numbness around the groin area in five patients. It disappeared the next day after surgery except in one patient with LCPD. This patient also showed complete recovery of the symptom at follow-up evaluation on the 7th day after surgery.

Table 2. Functional Outcomes of Hip Arthroscopy

\begin{tabular}{lcccc} 
Outcome measurement & Preoperative & Last follow-up & Proportion of patients with improvement (\%) & $p$-value \\
\hline VAS & $4.4 \pm 2.2$ & $1.9 \pm 2.1$ & 88.2 & $<0.001$ \\
\hline Modified HHS & $77.8 \pm 16.1$ & $90.0 \pm 17.1$ & 85.3 & 85.3 \\
WOMAC & $16.4 \pm 14.6$ & $6.8 \pm 12.5$ & 97.1 & $<0.001$ \\
\hline Hip joint flexion $\left({ }^{\circ}\right)$ & $101.7 \pm 19.0$ & $115.0 \pm 8.0$ & $<0.001$ \\
\hline
\end{tabular}

Values are presented as mean \pm standard deviation.

VAS: visual analog scale, HHS: Harris hip score, WOMAC: Western Ontario and McMaster Universities Osteoarthritis Index 


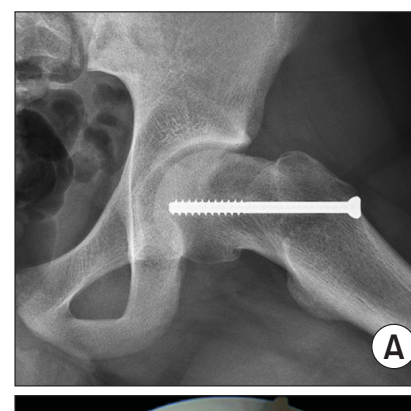

(A)
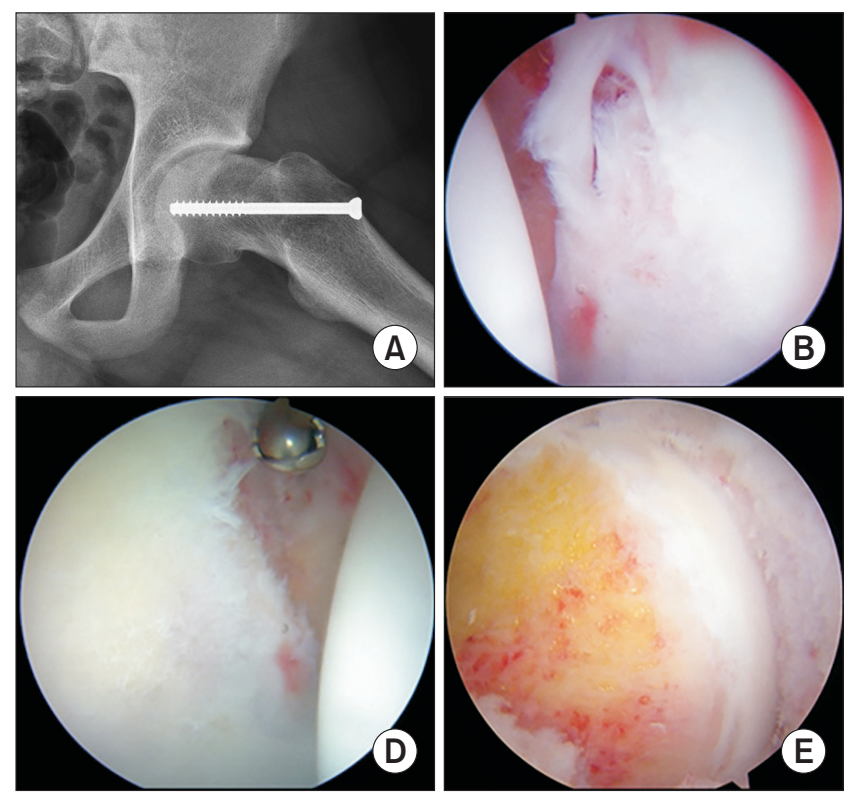

\section{DISCUSSION}

Hip arthroscopy was initially considered a challenging procedure because of deep location of the hip joint, limited visualization, need for traction, and potential for iatrogenic injury. ${ }^{7)}$ In children and adolescents, the small size and anatomical variability of the hip joint according to the developmental status, underlying disorders, and previous treatment may further raise safety issues. However, our results show that hip arthroscopy can be performed safely in skeletally immature patients. Complications of hip arthroscopy reported in the literature include neurapraxia, iatrogenic chondral damage, femoral neck fracture, fluid extravasation, and heterotopic ossification. ${ }^{12,13)}$ In our patients, there were no such complications except transient compression neuropathy of the perineal nerve. In addition, arthroscopic osteochondroplasty at the head-neck junction did not cause physeal growth disturbance even though patients were skeletally immature at the time of hip arthroscopy and the youngest patient in our series was 12.2 years old. According to literature, hip arthroscopy with traction of the affected leg can be safely performed in children with LCPD as young as 7 years old. ${ }^{14-16)}$

There are only a few studies on hip arthroscopic findings in LCPD. Synovial proliferation in the acetabular fossa and over the inner wall of the joint capsule is the most prominent finding in the early stage. ${ }^{16)}$ Arthroscopic findings in the late stage include a labral tear, ligamentum teres tear, osteochondritis dissecans, loose body, and impingement of the deformed femoral bump. ${ }^{17,18)}$ These pathologic changes in the late stage are believed to be

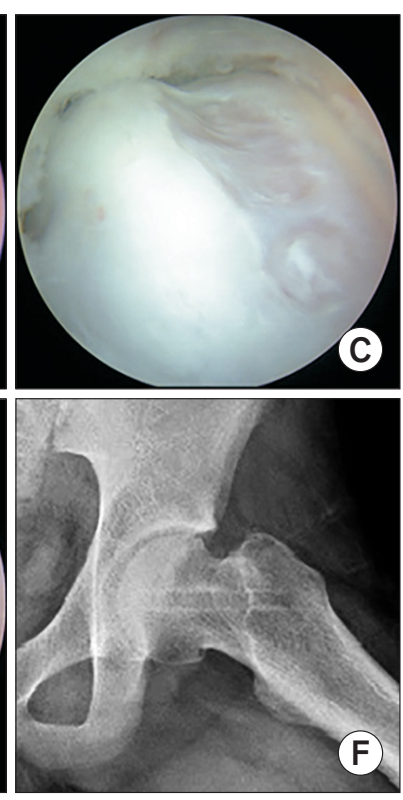

Fig. 1. A 15-year-old boy who underwent in situ screw fixation for mild to moderate slipped capital femoral epiphysis two years ago. A preoperative plain radiograph (A) showing decreased head-neck offset. Arthroscopic findings: torn labrum (B) and large femoral bump (C) impinging against the acetabular rim. Arthroscopic labral debridement (D) and osteochondroplasty (E) were performed. A postoperative plain radiograph (F) showing increased heckneck offset.

caused by abnormal mechanics between the deformed femoral head and acetabulum. ${ }^{17,18)}$ Arthroscopic treatments such as loose body removal, debridement of osteochondritis dissecans lesion, ${ }^{8)}$ and osteochondroplasty have been reported effective for improving pain and function. ${ }^{19)}$ All our patients were in the late reossification stage or residual stage. Their pathologic changes were mainly caused by impingement of the deformed femoral head against the acetabular margin. Labral tear was also present in five of six patients. Arthroscopic osteochondroplasty and labral debridement were effective in relieving symptoms in this condition. Because a spherical femoral head could not be created in any case due to the severe femoral head deformity, we performed labral debridement instead of repair.

Hip arthroscopy has been performed for pain management in hip dysplasia of skeletally immature patients. Fujii et al. ${ }^{20)}$ have reported intra-articular findings in symptomatic adolescent hip dysplasia: degeneration of the acetabular cartilage in $77.8 \%$ and a labral tear in $77.8 \%$. Ross et $\mathrm{al}^{21)}$ have also reported that $63 \%$ of cases have pathologic changes in the central compartment of the acetabulum and that more severe chondrolabral pathology is associated with severe dysplasia (center-edge angle, $<15^{\circ}$; acetabular index, $>20^{\circ}$ ). Dorrel and Catterall ${ }^{22)}$ did not recommend arthroscopic treatment for labral tears if the center-edge angle was less than $20^{\circ}$. These findings of previous reports correspond well with those of the present study. More than half of our patients had degenerative changes of the articular cartilage of the acetabulum. Arthroscopic treatment did not improve symptom in two hips with severe dysplasia. In severe dysplasia, we agree with the notion that 
Lim et al. Hip Arthroscopy for Pediatric and Adolescent Hip Disorders

Clinics in Orthopedic Surgery • Vol. 12, No. 1, $2020 \bullet$ www.ecios.org

arthroscopic labral debridement would not improve pain and that periacetabular osteotomy with or without proximal femoral osteotomy would be a definitive treatment. ${ }^{22}$

Most patients with SCFE have a risk of FAI due to post-slip deformity of the head-neck junction. ${ }^{23)}$ This deformity could lead to erosion of the anterolateral acetabular labrum and anterosuperior acetabular cartilage. ${ }^{24)}$ Treatment options for FAI in SCFE patients include modified Dunn procedure, flexion intertrochanteric osteotomy of the femur, and arthroscopic osteochondroplasty. ${ }^{24-26)}$ Chen et al. ${ }^{27)}$ reported that $88 \%$ of 40 patients had successful results after arthroscopic osteochondroplasty. In our practice, we perform arthroscopic osteochondroplasty for post-SCFE deformity only when the deformity is caused by mild to moderate slip. When the deformity is caused by severe slip, we prefer realignment osteotomy combined with osteochondroplasty through surgical hip dislocation approach to arthroscopic osteochondroplasty.

This study has some limitations. First, it is a retrospective case series without control group. Second, the follow-up period was short and various despite all skeletally immature patients reached skeletal maturity at the time of evaluation. Third, the modified HHS has not been validated for use in children or adolescents.

In summary, our short-term follow-up evaluation shows that hip arthroscopy is a safe procedure even in skeletally immature patients. It appears to be effective in improving functional impairment caused by FAI between the deformed femoral head and acetabulum or some intraarticular focal problems in pediatric and adolescent hip disorders. However, it does not mean that hip arthroscopy can prevent or delay the development of osteoarthritis. Further studies with a longer follow-up are necessary to determine how long the improved functional outcomes could last.

\section{CONFLICT OF INTEREST}

No potential conflict of interest relevant to this article was reported.

\section{REFERENCES}

1. Burman MS. Arthroscopy or the direct visualization of joints: an experimental cadaver study: 1931. Clin Orthop Relat Res. 2001;(390):5-9.

2. Byrd JW. Hip arthroscopy. J Am Acad Orthop Surg. 2006; 14(7):433-44.

3. Khanduja V, Villar RN. Arthroscopic surgery of the hip: current concepts and recent advances. J Bone Joint Surg Br. 2006;88(12):1557-66.

4. Lee YK, Moon KH, Kim JW, Hwang JS, Ha YC, Koo KH. Remaining loose bodies after arthroscopic surgery including extensive capsulectomy for synovial chondromatosis of the hip. Clin Orthop Surg. 2018;10(4):393-7.

5. Gross RH. Arthroscopy in hip disorders in children. Orthop Rev. 1977;6(9):43-9.

6. Kelly BT, Williams RJ 3rd, Philippon MJ. Hip arthroscopy: current indications, treatment options, and management issues. Am J Sports Med. 2003;31(6):1020-37.

7. Kocher MS, Kim YJ, Millis MB, et al. Hip arthroscopy in children and adolescents. J Pediatr Orthop. 2005;25(5):6806.

8. DeAngelis NA, Busconi BD. Hip arthroscopy in the pediatric population. Clin Orthop Relat Res. 2003;(406):60-3.

9. Berend KR, Vail TP. Hip arthroscopy in the adolescent and pediatric athlete. Clin Sports Med. 2001;20(4):763-78.
10. Stulberg SD, Cooperman DR, Wallensten R. The natural history of Legg-Calve-Perthes disease. J Bone Joint Surg Am. 1981;63(7):1095-108.

11. Southwick WO. Osteotomy through the lesser trochanter for slipped capital femoral epiphysis. J Bone Joint Surg Am. 1967;49(5):807-35.

12. Elsaidi GA, Ruch DS, Schaefer WD, Kuzma K, Smith BP. Complications associated with traction on the hip during arthroscopy. J Bone Joint Surg Br. 2004;86(6):793-6.

13. Clarke MT, Arora A, Villar RN. Hip arthroscopy: complications in 1054 cases. Clin Orthop Relat Res. 2003;(406):84-8.

14. Freeman CR, Jones K, Byrd JW. Hip arthroscopy for LeggCalve-Perthes disease: minimum 2-year follow-up. Arthroscopy. 2013;29(4):666-74.

15. Kuklo TR, Mackenzie WG, Keeler KA. Hip arthroscopy in Legg-Calve-Perthes disease. Arthroscopy. 1999;15(1):88-92.

16. Suzuki S, Kasahara Y, Seto Y, Futami T, Furukawa K, Nishino Y. Arthroscopy in 19 children with Perthes' disease: pathologic changes of the synovium and the joint surface. Acta Orthop Scand. 1994;65(6):581-4.

17. Roy DR. Arthroscopic findings of the hip in new onset hip pain in adolescents with previous Legg-Calve-Perthes disease. J Pediatr Orthop B. 2005;14(3):151-5.

18. Ross JR, Nepple JJ, Baca G, Schoenecker PL, Clohisy JC. In- 
Lim et al. Hip Arthroscopy for Pediatric and Adolescent Hip Disorders

Clinics in Orthopedic Surgery • Vol. 12, No. 1, $2020 \bullet$ www.ecios.org

traarticular abnormalities in residual Perthes and Perthes-like hip deformities. Clin Orthop Relat Res. 2012;470(11):296877.

19. Snow SW, Keret D, Scarangella S, Bowen JR. Anterior impingement of the femoral head: a late phenomenon of LeggCalve-Perthes' disease. J Pediatr Orthop. 1993;13(3):286-9.

20. Fujii M, Nakashima Y, Jingushi S, et al. Intraarticular findings in symptomatic developmental dysplasia of the hip. J Pediatr Orthop. 2009;29(1):9-13.

21. Ross JR, Zaltz I, Nepple JJ, Schoenecker PL, Clohisy JC. Arthroscopic disease classification and interventions as an adjunct in the treatment of acetabular dysplasia. Am J Sports Med. 2011;39 Suppl:72S-78S.

22. Dorrell JH, Catterall A. The torn acetabular labrum. J Bone Joint Surg Br. 1986;68(3):400-3.
23. Roy DR. The use of hip arthroscopy in the management of the pediatric hip. J Hip Preserv Surg. 2016;3(2):97-107.

24. Rab GT. The geometry of slipped capital femoral epiphysis: implications for movement, impingement, and corrective osteotomy. J Pediatr Orthop. 1999;19(4):419-24.

25. Parsch K, Zehender H, Buhl T, Weller S. Intertrochanteric corrective osteotomy for moderate and severe chronic slipped capital femoral epiphysis. J Pediatr Orthop B. 1999;8(3):223-30.

26. Clohisy JC, Zebala LP, Nepple JJ, Pashos G. Combined hip arthroscopy and limited open osteochondroplasty for anterior femoroacetabular impingement. J Bone Joint Surg Am. 2010;92(8):1697-706.

27. Chen A, Youderian A, Watkins S, Gourineni P. Arthroscopic femoral neck osteoplasty in slipped capital femoral epiphysis. Arthroscopy. 2014;30(10):1229-34. 